\section{Verbal processing in third-grade children}

\author{
KENNETH A. JACOBUS, University of \\ Georgia, Athens, Ga. 30601
}

Johnson's (1968) decoding-operation model proposes a way in which Ss learn and recall verbal material. The model has been quite successful in predicting the performance of adults in a paired-associate task. The present study examined the extent of fit between the model and performance of third graders. The model was partially supported.

Johnson's (1968) decoding-operation model proposes a way in which Ss learn and recall verbal responses. The model has been quite successful in predicting the performance of adults in paired-associate (PA) task situations (Johnson, 1968). The present study was concerned with examining the value of the model for predicting performance of third-grade children.

$A$ basic assumption of the model is that $\mathrm{Ss}$ divide the elements of a response into chunks (Miller, 1956) which are organized hierarchically and stored in memory as a single code. Recall is assumed to follow a specified sequence of descending decoding operations through the hierarchical structure. Specifically, when the stimulus associated with a learned response is presented, Ss recall the code representing the entire response, then decode down through the chunk hierarchy, translating each of the codes into its components. All but the first component is stored in short-term memory (STM), while the first component is decoded into its components. If, when a code is decoded into its components, the first component of the code is a nonreducible response element, it is produced overtly, and $S$ then retrieves the next element from STM.

The model can be illustrated as follows: Assume $S$ is recalling a response such as DHMZ which he has organized into two chunks, DH and MZ. Johnson's decoding model would propose that $S$ would proceed through the following decoding steps: (1) recall the code representing the entire response and decode it into its elements (the code for $\mathrm{DH}$ and the code for $\mathrm{MZ}$ ); (2) decode the "DH" code into its elements (D and $\mathrm{H}$ ); (3) overtly produce $\mathrm{D}$; (4) overtly produce $\mathrm{H}$; (5) decode the "MZ" code into its elements; (6) overtly produce $\mathrm{M}$;(7) overtly produce $Z$.

One implication of the model is that more decoding operations are performed at the point between chunks than at points within chunks. One way of examining this implication (Johnson, 1968) is to assume that Ss will terminate in their response attempt where they become uncertain, and the probability of becoming uncertain is a function of the number of decoding operations which must be performed at a response point. More specifically, Johnson has measured the performance at each response point using the TEP stop error which is the probability of going from a correct recall to a stop error within a response. According to the above assumptions, the model can make two predictions: (1) the TEP stop error at between-chunk points should be significantly higher than at within-chunk points, and (2) the TEP stop error at a between-chunk point should increase as the size of the chunk which follows the between-chunk point increases.

The predictions were tested with adults in a PA learning study (Johnson, 1968), where each response was a sequence of letters (e.g., DH VQ) with a blank appearing between the second and third letters. It was assumed that Ss would use the blank space as a means of organizing the response into chunks, and the data supported these assumptions. The TEP stop error at the blank was higher than at within-chunk points, and the TEP stop error at the blank increased as the size of chunk following the blank increased.

The present study was designed to determine the extent of fit between the decoding model and behavior of third-grade children in a similar learning-recall situation.

\section{METHOD}

The Ss were 50 third-grade children ( 27 males and 23 females) from Oglethorpe County Elementary School, Oglethorpe County, Georgia.

Two PA lists were constructed, each list having the digits 1 to 4 as stimuli and with responses of four-letter (List 1) or five-letter (List 2) sequences. In both lists, a blank appeared between the second and third letters of each response (e.g., NG VH and NG VHS). Each response had low associative frequencies between successive letters (Underwood \& Schulz, 1960) and no letter was repeated more than once with in a list.

Separate groups of Ss learned Lists 1 and 2 by the standard anticipation technique for 15 trials (one study and 14 learning). If $S$ did not respond to a stimulus during a response-recall phase within $5 \mathrm{sec}$, the $\mathrm{E}$ presented the response. During each presentation of a response, $S$ was asked to read aloud each letter of the response. The stimuli and responses were typewritten on $3 \times 5$ in. white cards and presented to Ss by hand. Five randomizations of PA order were used for each list.

The mean TEP stop error was computed for each response point. The TEP stop error is based on the ratio of the total number of stop errors for which the preceding letter is recalled correctly to the total number of times for which that letter is recalled correctly. If the $E$ could not compute a TEP stop error at each response point for an $S$, the $S$ was not used in the analysis. As a result of this procedure, one $S$ in each list group was not used.

\section{RESULTS}

The mean number of correct responses during learning for the List 1 and 2 groups was 9.21 and 8.83 , respectively $[t(46)=.45$, $p>.05]$

The point between the first and second letter of a response was designated Point 1 ; the point between the second and third letter (i.e., the blank space) was designated Point 2; and so on. For List 1, the mean TEP stop errors for Points 1,2 , and 3 were .01 , .13 , and .02 , respectively $[F(2,46)=11.11$, $p<.05]$; for List 2, the mean TEP stop errors for Points $1,2,3$, and 4 were .02,.19, .04 , and .02 , respectively $[\mathrm{F}(3,69)=30.91$, $\mathrm{p}<.05$ ] . The Neuman-Keuls (Winer, 1962) procedure was used to test which of the differences within each type of response were significant. For both lists, the TEP stop error at the blank was significantly higher $(p<.05)$ than at other points within the response; for both lists, no other differences were significant $(p>.05)$.

The influence of the size of the second chunk (List 1 vs List 2) on the TEP stop errors at Points 1, 2, and 3 was examined. For Point 1, $t(46)=1.26, p>.05$; for Point 2, $t(46)=1.36, p>.05$; and for Point $3, \mathrm{t}(46)=1.17, \mathrm{p}>.05$.

\section{DISCUSSION}

The results give some support for the decoding-operation model. As predicted, the TEP stop error was significan tly higher at the blank for Lists 1 and 2 than at other response points. In addition, the TEP stop error at the blank increased as the size of the chunk which followed the blank increased. This increase, however, was not statistically significant. These results suggest a need for additional research with children in similar learning-recall task situations.

\section{REFERENCES}

JOHNSON, N. F. Sequential verbal behavior. In T. Dickson and D. Horton (Eds.), Verbal behavior and general behavior theory. Englewood Cliffs, N.J.: Prentice-Hall, 1968.

MILLER, G. The magical number seven, plus or minus two: Some limits on our capacity for processing information. Psychological Review, 1956, 69, 81-97.

UNDERWOOD, B. J., \& SCHULZ, R. W. Meaningfulness and verbal learning. Chicago: Lippincott, 1960.

WINER, B. Statistical principles in experimental design. New York: McGraw-Hill, 1962. 